Dochead fiche

Sous-dochead juridique

\title{
Délivrance des médicaments stupéfiants
}

\section{Mathieu Guerriaud}

Maître de conférences en droit pharmaceutique et de la santé, pharmacovigilance et iatrogénie Université de Bourgogne Franche-Comté, UFR des sciences de santé/CREDIMI, 7 boulevard Jeanned'Arc, 21079 Dijon, France

Adresse e-mail : mathieu.guerriaud@u-bourgogne.fr (M. Guerriaud).

\section{Résumé}

La délivrance des médicaments stupéfiants à l'officine est une opération complexe comportant beaucoup d'étapes et s'entourant de règles qu'il est important de connaître. Ce cadre, qui peut apparaître contraignant, a été mis en place pour protéger la société des dangers que représentent ces médicaments.

(C) 2019

Mots clés - délivrance ; médicament stupéfiant ; ordonnance sécurisée ; sécurité sanitaire

La délivrance des médicaments stupéfiants à l'officine repose en premier lieu sur une analyse réglementaire de l'ordonnance en vue de vérifier si le prescripteur a le droit de prescrire un médicament stupéfiant, si l'ordonnance est valable au regard de la date de prescription, si elle est sécurisée et correctement rédigée. À la suite de cette analyse, une succession de tâches administratives doit être réalisée.

\section{T1 Prescripteurs habilités}

L'article R. 5132-6 du Code de la santé publique (CSP) énumère les professionnels pouvant prescrire des médicaments classés comme stupéfiants sur prescription ou commande à usage professionnel (tableau 1) [1]. 
TEG1 Le médecin dispose d'une grande liberté de prescription [2,3]. II peut choisir la thérapeutique la plus adaptée pour son patient ou son usage professionnel. La prescription de quelques médicaments stupéfiants est toutefois réservée à certains médecins. C'est le cas de la méthadone :

- la prescription initiale de la forme gélule est réservée aux médecins exerçant en centres de soins d'accompagnement et de prévention en addictologie (CSAPA) ;

- celle de la forme sirop est étendue aux médecins hospitaliers dans un contexte d'hospitalisation, de consultation ou en milieu pénitentiaire.

TEG1 Le chirurgien-dentiste peut prescrire tous les actes, produits et prestations nécessaires à l'exercice de l'art dentaire [4,5], dont des médicaments stupéfiants pour ses patients ou son usage professionnel.

TEG1 La sage-femme subit une double limitation : elle ne peut prescrire que certains médicaments et produits [6] et uniquement aux patients auxquels elle apporte des soins (une femme ou son enfant) [7]. Parmi les médicaments stupéfiants, il ne lui est possible de prescrire que le chlorhydrate de morphine sous forme d'ampoules injectables à $10 \mathrm{mg}$, dans la limite de deux ampoules par patiente ou pour son usage professionnel.

TEG1 Le vétérinaire dispose d'un droit de prescription en ce qui concerne la médecine vétérinaire, droit très étendu puisqu'il peut, dans certains cas, prescrire des médicaments humains, dont les stupéfiants.

Tableau 1. Les prescripteurs de médicaments stupéfiants.

\begin{tabular}{lll}
\hline Prescripteurs & Restrictions & Type de médicaments \\
\hline Médecin & Aucune & $\begin{array}{l}\text { Tous les médicaments stupéfiants à usage } \\
\text { humain }\end{array}$ \\
\hline $\begin{array}{l}\text { Chirurgien- } \\
\text { dentiste }\end{array}$ & Limité à l'art dentaire & $\begin{array}{l}\text { Tous les médicaments stupéfiants à usage } \\
\text { humain dans le cadre de l'art dentaire } \\
\text { (pas la méthadone) }\end{array}$ \\
\hline Sage-femme & Femme et son enfant & $\begin{array}{l}\text { Uniquement le chlorhydrate de morphine sous } \\
\text { forme d'ampoules injectables à 10 mg, dans } \\
\text { la limite de deux ampoules par patiente }\end{array}$ \\
& & $\begin{array}{l}\text { Tous les médicaments stupéfiants à usage } \\
\text { vétérinaire et, si besoin, les médicaments } \\
\text { stupéfiants à usage humain }\end{array}$ \\
\hline Vétérinaire & Aucune, mais chez l'animal &
\end{tabular}

Sur 2 colonnes ou 1 colonne + marge 


\section{T1 Date de l'ordonnance}

TEG1 II est interdit de prescrire un médicament stupéfiant pour une durée de traitement supérieure à 28 jours [8], durée qui peut être réduite à sept ou quatorze jours (tableau 2). Un fractionnement de délivrance peut également être exigé.

TEG1 L'ordonnance doit être présentée dans les trois jours suivant sa rédaction (délai de carence) [9]. Au-delà, les médicaments ne sont délivrés que pour la durée restant à courir.

TEG1 II faut également être vigilant au chevauchement des ordonnances de stupéfiants : "Une nouvelle ordonnance ne peut être ni établie ni exécutée par les mêmes praticiens pendant la période déjà couverte par une précédente ordonnance prescrivant de tels médicaments, sauf si le prescripteur en décide autrement par une mention expresse portée sur l'ordonnance " [9]. En pratique, pour délivrer la deuxième ordonnance, il faut décompter les médicaments couverts par la première.

Tableau 2. Médicaments stupéfiants à délais particuliers.

\begin{tabular}{lll}
\hline Stupéfiants & Fractionnement & $\begin{array}{l}\text { Durée maximale de } \\
\text { prescription }\end{array}$ \\
\hline Chlorhydrate de méthadone & 7 jours & 14 jours (solution buvable)
\end{tabular}

\begin{tabular}{|c|c|c|}
\hline Méthadone APHP ${ }^{\circledR}$ & 7 jours & 28 jours (gélule) \\
\hline $\begin{array}{l}\text { Chlorhydrate de morphine } \\
\text { injectable }\end{array}$ & 7 jours & $\begin{array}{l}28 \text { jours en cas } \\
\text { d'administration à l'aide d'un } \\
\text { système actif pour perfusion }\end{array}$ \\
\hline $\begin{array}{l}\text { Chlorhydrate de } \\
\text { péthidine (Péthidine }{ }^{\circledR} \text { ) }\end{array}$ & & 7 jours \\
\hline $\begin{array}{l}\text { Citrate de fentanyl (Abstral }^{\circledR} \text {, } \\
\text { Actiq }^{\circledR}, \text { Breakyl }^{\circledR}, \text { Effentora }^{\circledR} \text {, } \\
\text { Instanyl }^{\circledR}, \text { Pecfent }^{\circledast}, \text { Recivit }^{\circledast} \text { ) }\end{array}$ & 7 jours & 28 jours \\
\hline Fentanyl (Durogésic ${ }^{\circledR}$ ) & 14 jours & 28 jours \\
\hline Sulfate de morphine injectable & 7 jours & $\begin{array}{l}28 \text { jours en cas } \\
\text { d'administration à l'aide d'un } \\
\text { système actif pour perfusion }\end{array}$ \\
\hline
\end{tabular}




\section{T1 Ordonnance sécurisée}

TEG1 La prescription de médicaments stupéfiants doit être rédigée sur une ordonnance sécurisée [10], dont les particularités sont les suivantes :

- caducée médical en filigrane sur papier blanc naturel sans azurant optique ;

- mentions préimprimées en bleu ;

- numérotation du lot d'ordonnances ;

- carré de sécurité en microlettres dans lequel le praticien inscrit le nombre de médicaments (spécialité ou préparation) prescrits.

TEG1 Certaines mentions sont obligatoires sur cette ordonnance (tableau 3) sur laquelle le prescripteur doit apposer sa signature immédiatement sous la dernière ligne ou rendre inutilisable l'espace laissé libre entre cette dernière ligne et sa signature par tout moyen approprié [11].

Tableau 3. Mentions obligatoires sur une ordonnance de stupéfiants.

\begin{tabular}{|c|c|c|}
\hline $\begin{array}{l}\text { Informations concernant } \\
\text { le prescripteur }\end{array}$ & $\begin{array}{l}\text { Informations concernant } \\
\text { le patient }\end{array}$ & $\begin{array}{l}\text { Informations concernant } \\
\text { le traitement }\end{array}$ \\
\hline Nom et prénoms & Nom et prénoms & $\begin{array}{l}\text { Date à laquelle l'ordonnance } \\
\text { a été rédigée }\end{array}$ \\
\hline Qualité ou titre ou spécialité & Sexe & \\
\hline Identifiant (Adeli ou RPPS) & Date de naissance du malade & $\begin{array}{l}\text { DCl du médicament } \\
\text { ou du produit prescrit }\end{array}$ \\
\hline $\begin{array}{l}\text { Adresse professionnelle } \\
\text { précisant la mention "France" }\end{array}$ & Taille et poids (si nécessaire) & $\begin{array}{l}\text { Posologie et mode d'emploi : } \\
\text { rédaction en toutes lettres }\end{array}$ \\
\hline $\begin{array}{l}\text { Coordonnées téléphoniques } \\
\text { précédées de l'indicatif } \\
\text { international "+33" }\end{array}$ & & $\begin{array}{l}\text { thérapeutiques par prise, } \\
\text { nombre de prises, dosage s'il } \\
\text { s'agit de spécialités, doses ou }\end{array}$ \\
\hline Adresse électronique & & $\begin{array}{l}\text { concentrations de substances } \\
\text { et nombre d'unités ou volume }\end{array}$ \\
\hline $\begin{array}{l}\text { Médicaments à prescription } \\
\text { hospitalière ou à prescription } \\
\text { initiale hospitalière : nom de } \\
\text { l'établissement ou du service } \\
\text { de santé }\end{array}$ & & s'il s'agit de préparations \\
\hline
\end{tabular}




\section{T1 Opérations à réaliser à l’officine}

TEG1 Pour assurer une traçabilité maximale de la délivrance, un certain nombre d'opérations doivent être réalisées à la suite de l'analyse réglementaire de l'ordonnance.

TEG1 Toute entrée et toute sortie de substances et de médicaments classés comme stupéfiants sont inscrites sur le registre comptable des stupéfiants qui peut être manuscrit ou électronique.

TEG1 La transcription au registre suit les règles classiques édictées à l'article R. 5132-10 du CSP [12]. Le pharmacien enregistre le nom et l'adresse du porteur de l'ordonnance lorsque celui-ci n'est pas le malade, qui doit, s'il est inconnu de l'officine, présenter une pièce d'identité dont les références seront reportées sur le registre [13].

TEG1 Après exécution, certaines mentions sont apposées sur l'ordonnance ou le bon de commande [14] : timbre de l'officine, numéro(s) d'enregistrement, date d'exécution, quantités délivrées formulées en unités de prise et non pas en nombre de boîtes [13], mentions relevant de la substitution d'un princeps.

TEG1 Sur les boîtes de médicaments, le pharmacien inscrit la posologie, son nom, son adresse et le numéro d'enregistrement au registre dans le cadre entouré d'un filet rouge [13].

TEG1 Une copie de toutes les ordonnances comportant la prescription d'un ou plusieurs médicaments classés comme stupéfiants ou soumis à la réglementation des stupéfiants doit être conservée à l'officine pendant une durée de trois ans [13].

Déclaration de liens d'intérêts

L'auteur déclare ne pas avoir de liens d'intérêts.

\section{Références}

[1] Code de la santé publique. Article R. 5132-6.

www.legifrance.gouv.fr/affichCodeArticle.do?cidTexte=LEGITEXT000006072665\&idArticle=LEGIARTIO 00006915538\&dateTexte $=\&$ categorieLien $=c i d$

[2] Code de la santé publique. Article R. 4127-8.

www.legifrance.gouv.fr/affichCodeArticle.do?cidTexte=LEGITEXT000006072665\&idArticle=LEGIARTIO 00006912869 
[3] Code de la sécurité sociale. Article L. 162-2

www.legifrance.gouv.fr/affichCodeArticle.do;?idArticle=LEGIARTI000006741332\&cidTexte=LEGITEXT 000006073189

[4] Code de la santé publique. Article L. 4141-1.

www.legifrance.gouv.fr/affichCodeArticle.do?idArticle=LEGIARTI000018899595\&cidTexte=LEGITEXTO 00006072665\&dateTexte $=20080601$

[5] Code de la santé publique. Article L. 4141-2.

www.legifrance.gouv.fr/affichCodeArticle.do?cidTexte=LEGITEXT000006072665\&idArticle=LEGIARTIO 00006688895\&dateTexte=\&categorieLien $=$ cid

[6] Code de la santé publique. Article L. 4151-4.

www.legifrance.gouv.fr/affichCodeArticle.do?idArticle=LEGIARTI000006688934\&cidTexte=LEGITEXTO 00006072665\&dateTexte $=20081204$

[7] Arrêté du 12 octobre 2011 fixant la liste des médicaments que peuvent prescrire les sagesfemmes et portant abrogation de dispositions réglementaires. www.legifrance.gouv.fr/affichTexte.do?cidTexte=JORFTEXT000024686131\&categorieLien=id

[8] Code de la santé publique. Article R. 5132-30.

www.legifrance.gouv.fr/affichCodeArticle.do?idArticle=LEGIARTI000006915579\&cidTexte=LEGITEXTO 00006072665\&dateTexte $=20081204$

[9] Code de la santé publique. Article R. 5132-33.

www.legifrance.gouv.fr/affichCodeArticle.do?idArticle=LEGIARTI000006915586\&cidTexte=LEGITEXTO 00006072665\&dateTexte $=20081204$

[10] Code de la santé publique. Article R. 5132-5.

www.legifrance.gouv.fr/affichCodeArticle.do?cidTexte=LEGITEXT000006072665\&idArticle=LEGIARTIO 00006915537\&dateTexte=\&categorieLien=cid

[11] Code de la santé publique. Article R. 5132-4.

www.legifrance.gouv.fr/affichCodeArticle.do?cidTexte=LEGITEXT000006072665\&idArticle=LEGIARTIO 00006915535\&dateTexte=\&categorieLien $=$ cid

[12] Code de la santé publique. Article R. 5132-10.

www.legifrance.gouv.fr/affichCodeArticle.do?idArticle=LEGIARTI000018042965\&cidTexte=LEGITEXTO 00006072665\&dateTexte $=20090112$

[13] Code de la santé publique. Article R. 5132-35.

www.legifrance.gouv.fr/affichCodeArticle.do?idArticle=LEGIARTI000006915589\&cidTexte=LEGITEXTO $00006072665 \&$ dateTexte $=20081204$

[14] Code de la santé publique. Article R. 5132-13.

www.legifrance.gouv.fr/affichCodeArticle.do?cidTexte=LEGITEXT000006072665\&idArticle=LEGIARTIO $00006915552 \&$ dateTexte $=\&$ categorieLien $=$ cid 